\title{
THE DUAL NEST FOR DEGENERATE YOCCOZ PUZZLES
}

\author{
MAGNUS ASPENBERG
}

\begin{abstract}
The Yoccoz puzzle is a fundamental tool in Holomorphic Dynamics. The original combinatorial argument by Yoccoz, based on the BrannerHubbard tableau, counts the preimages of a non-degenerate annulus in the puzzle. However, in some important new applications of the puzzle (notably, matings of quadratic polynomials) there is no non-degenerate annulus. We develop a general combinatorial argument to handle this situation. It allows us to derive corollaries, such as the local connectedness of the Julia set, for suitable families of rational maps.
\end{abstract}

\section{INTRODUCTION}

The Yoccoz puzzle is by now well known in Complex Dynamics, as a way to prove local connectivity of Julia sets for non-renormalisable (or finitely renormalisable) quadratic polynomials. The result was proven in 1990 by J.-C. Yoccoz (see [3], 7]), using a tableau developed by Branner and Hubbard [2. Other approaches have been developed also for some infinitely renormalisable polynomials (see e.g. 6], [4, 5], etc). The set of puzzle pieces is a dynamical partition of a neighbourhood of the Julia set for some function, which in the original setting was a quadratic polynomial of the form $f_{c}(z)=z^{2}+c, c \in \mathbb{C}$. Each puzzle piece is simply connected and contains a connected part of the Julia set of $f_{c}$. Hence, by definition, if the puzzle pieces shrink to points, then the Julia set must be locally connected at that point. Two things must be satisfied for this to happen: Firstly, the puzzle must have a good combinatorics. This property is inherited from the fact that $f_{c}$ is non-renormalisable. Secondly, nested puzzle pieces must not touch each other, i.e. there must be a non-degenerate annulus between two puzzle pieces in the BrannerHubbard tableau.

In the case of quadratic polynomials, this non-degeneracy is never a problem, because in this case the puzzle pieces are formed by equipotentials and external rays which behave nicely. However, the situation with degenerate annuli appears naturally in more general applications of the Yoccoz puzzle, in particular for rational functions, the reason being that the corresponding equipotentials and external rays for rational maps have a more complicated structure than for quadratic polynomials (see e.g. [1]). Already for higher degree polynomials, the problem of degenerate annuli appears (see e.g. [1], [9], 10]).

Received by the editors April 8, 2009.

2000 Mathematics Subject Classification. Primary 37F20; Secondary 30D05.

The author gratefully acknowledges funding from the Research Training Network CODY of the European Commission.

(C)2009 American Mathematical Society Reverts to public domain 28 years from publication 
In this paper we give a method of how to deal with this degeneracy problem in a consistent way. We show that if one has a Yoccoz puzzle with "good combinatorics" but without the non-degeneracy condition, then the puzzle pieces still shrink to points. The idea is to look at the space between degenerate annuli (complementary annuli) and compute their total modulus given that the combinatorics is correct.

1.1. Abstract statement of result. We first state the main result in a combinatorial and somewhat abstract way. In Section 2 we discuss the Yoccoz puzzle and from this it will be clear where these abstract statements come from. In Section 3 we prove Theorem $\mathrm{A}$ and in Section 4 we discuss some applications.

A degenerate annulus will be understood as the closed set bounded by two closed Jordan arcs $\gamma_{1}$ and $\gamma_{2}$, which touch each other in at least one point. We assume the interior is non-empty. A non-degenerate annulus is simply an annulus with positive modulus. For an annulus $A$ (degenerate or non-degenerate) we say that the inner component $\operatorname{in}(A)$ of $A$ is the bounded component of $A^{c}$ and the outer component $\operatorname{out}(A)$ of $A$ is the unbounded component of $A^{c}$. We say that a sequence of annuli $A_{j}$ is a-nested if $i n\left(A_{j+1}\right) \cup A_{j+1} \subset i n\left(A_{j}\right)$ for all $j \geq 0$. Of course the $A_{j}$ has to be disjoint if they are a-nested. We say that an annulus $A$ surrounds a set $E$ if $E \subset i n(A)$. Given an analytic function $f$, a sequence of annuli $A_{j}$ satisfies the Markov property if $f^{n}\left(A_{j}\right) \cap A_{i}=\emptyset$ unless $f^{n}\left(A_{j}\right)=A_{i}$, for all $i, j, n \geq 0$.

The setup is an a-nested sequence of annuli mapped onto each other in a certain unbranched way. In the following theorem, we say that $f: A \rightarrow B$ is a covering map between the closed annuli $A$ and $B$, if $f$ is a covering map on some neighbourhood of $A$ onto some neighbourhood of $B$. Here is the abstract formulation of the main result.

Theorem A. Suppose $A_{j}$ is an a-nested sequence of (not necessarily non-degenerate) annuli $A_{j}$ satisfying the Markov property and surrounding a critical point $z_{0}$ of order 2 of an analytic function $f$, having the following properties: For each $A_{j}$, $j>0$, there is some $A_{j^{\prime}}$ and $n_{j}>0$ such that

$$
f^{n_{j}}: A_{j} \rightarrow A_{j^{\prime}}
$$

is an unbranched covering of degree 2. Conversely, each $A_{j}$ has (at least) 2 preimages $A_{j_{1}}, A_{j_{2}}$, with $j_{1}, j_{2}>j$, such that

$$
f^{n_{j_{i}}}: A_{j_{i}} \rightarrow A_{j}, \quad i=1,2
$$

are both unbranched coverings of degree 2.

Finally, suppose that the complementary annuli $\alpha_{j}=\operatorname{in}\left(A_{j}\right) \cap \operatorname{out}\left(A_{j+2}\right), j=$ $0,1, \ldots$, are all non-degenerate. Then there is a sequence of non-overlappling $\alpha_{j_{k}}$, $k=0,1, \ldots$, such that

$$
\sum_{k} \bmod \left(\alpha_{j_{k}}\right)=\infty
$$

We call the set of complementary annuli a dual nest of annuli around the critical point. The reason for taking the space between every second annulus in the $a$-nested sequence $A_{j}$ instead of just $i n\left(A_{j}\right) \cap$ out $\left(A_{j+1}\right)$ is that the latter complementary annuli might be degenerate. However, the former annuli will always be non-degenerate as we shall see. 


\section{The Yoccoz Puzzle}

In this section let us recapitulate the idea of Yoccoz's famous result of proving that the Julia set of a non-renormalisable quadratic polynomial $f_{c}(z)=z^{2}+c$ is locally conneced. We will follow the exposition in [7]. The setup is a puzzle partition by B. Branner and J. Hubbard originally made for polynomials of degree 3. By utilising the combinatorics of the puzzle for a non-renormalisable polynomial, Yoccoz could then prove that these puzzles pieces shrink to points, thereby proving that the Julia set is locally connected.

So let $f_{c}(z)=z^{2}+c$ be non-renormalisable. It has 2 fixed points, of which at least one must be repelling. Let $\Psi: \hat{\mathbb{C}} \backslash \mathbb{D} \rightarrow \hat{\mathbb{C}} \backslash J(f)$ be the Böttcher coordinates around $\infty$. An external ray with angle $\theta$ is defined by

$$
\gamma(\theta)=\left\{z=r e^{2 \pi i \theta}: \Psi^{-1}\left(r e^{2 \pi i \theta}\right), r>1\right\} .
$$

An external ray with angle $\theta$ lands, i.e. the $\operatorname{limit}_{\lim } \lim _{r \rightarrow 1} \Psi^{-1}\left(r e^{2 \pi i \theta}\right)$ exists, when $\theta$ is a rational number. Evidently, landing points of such rays have to be periodic points. Conversely, every periodic point is a landing point of finitely many external rays with rational angles (although this is non-trivial to prove, see e.g. 8]).

The fixed point on which an external ray with angle $\theta \neq 0$ lands, is called the $\alpha$-fixed point. The other fixed point, where the external ray with angle 0 lands, is called the $\beta$-fixed point. An equipotential of level $r>1$ is defined by

$$
E(r)=\left\{z=r e^{2 \pi i \theta}: 0 \leq \theta \leq 2 \pi\right\} .
$$

Assume now that the rays $\gamma\left(\theta_{j}\right)$ land at the $\alpha$-fixed point. Take some $r_{0}>1$. Then these rays together with the equipotential of level $r_{0}$ form a puzzle (of depth zero) where each puzzle piece is a bounded component of the complement of $E\left(r_{0}\right) \cup$ $\bigcup_{j} \gamma\left(\theta_{j}\right)$. Since the set $\gamma\left(\theta_{j}\right)$ of external rays are forward invariant, we can pull back these puzzle pieces and thereby obtain the puzzle of depth one, consisting of the puzzles pieces so that each puzzle piece is a preimage of a puzzle piece of depth zero. If $P_{d}(z)$ is the puzzle piece of level $d$ containing $z$, then it follows by definition that $f_{c}\left(P_{d}(z)\right)=P_{d-1}\left(f_{c}(z)\right)$. We can also number the puzzle pieces of depth $d$ and write $P_{d}^{j}, j=0, \ldots, n-1$ where $n$ is the number of puzzles pieces of depth $d$. We usually denote by $P_{d}^{0}=P_{d}(0)$ the critical puzzle piece containing the critical point $z=0$. Because of the forward invariance we have the following immediate Markov property:

Lemma 2.1. Given two puzzle pieces $P$ and $Q$ either $P \cap Q=\emptyset$ or one is contained in the other.

Note that for each puzzle piece $P$ we have that $P \cap J\left(f_{c}\right)$ is connected. Hence if the puzzle pieces around a given point $z$ shrink to a single point, namely $z$, then the Julia set is locally connected at $z$.

To prove this we will study the annuli around the critical point $z=0$ :

$$
A_{d}(z)=P_{d}(z) \backslash \overline{P_{d-1}(z)} \text {. }
$$

Given an (open) annulus $A=P_{d}(0) \backslash \bar{P}_{d^{\prime}}(0)$ (where $d<d^{\prime}$ ) surrounding the critical point $z=0$ (i.e. the inner puzzle piece contains $z=0$ ) suppose it is subdivided into two (open) annuli $B$ and $C$ which also surround the critical point, i.e. $\bar{A}=\overline{B \cup C}$. Then we have the following Grötzsch inequality:

$$
\bmod (A) \geq \bmod (B)+\bmod (C) .
$$


Hence if $\sum_{d} A_{d}(0)=\infty$ we have that $\bigcap_{d} P_{d}(0)=\{0\}$.

Although Yoccoz's result is stated for quadratic polynomials, the ideas are mostly combinatorial and since we will aim for more general applications, we formulate his theorem in a more combinatorial way. But first we have to define the BrannerHubbard tableau. Given a point $z \in J\left(f_{c}\right)$, not in $\bigcup_{j \geq 0} f_{c}^{-j}(\alpha)$, where $\alpha$ is the $\alpha$-fixed point, we consider its orbit under $f_{c}$ :

$$
z \rightarrow z_{1} \rightarrow z_{2} \rightarrow \ldots
$$

where $f_{c}\left(z_{j}\right)=z_{j+1}$. For a given depth $d$, we say that $z_{j} \in P_{d}\left(z_{j}\right)$ is critical, semi-critical or off-critical whenever respectively:

- $z_{j} \in P_{d}(0)$, (critical)

- $z_{j} \in P_{d-1}(0) \backslash P_{d}(0)$, (semi-critical)

- $z_{j} \notin P_{d-1}(0)$. (off-critical)

Now note that if $z_{j} \in P_{d}(z)$, then the map $f_{c}: P_{d}\left(z_{j}\right) \rightarrow P_{d-1}\left(z_{j+1}\right)$ is either a double covering if $z_{j}$ is critical and univalent if $z_{j}$ is semi-critical or off-critical.

Moreover, in the critical and off-critical case, the map $f_{c}: A_{d}\left(z_{j}\right) \rightarrow A_{d-1}\left(z_{j+1}\right)$ is a covering map. In the semi-critical case it is not a covering map, since $P_{d}\left(z_{j+1}\right)$ has two preimages in $P_{d-1}\left(z_{j}\right)$, but we have the following relationships of the modulus of these annuli:

Lemma 2.2. With notation as above, we have:

$$
\begin{aligned}
& \bmod \left(A_{d-1}\left(z_{j}\right)\right)=2 \bmod \left(A_{d}\left(z_{j}\right)\right) \text { if } z_{j} \text { is critical, } \\
& \bmod \left(A_{d-1}\left(z_{j}\right)\right)=\bmod \left(A_{d}\left(z_{j}\right)\right) \text { if } z_{j} \text { is off-critical, and } \\
& \bmod \left(A_{d-1}\left(z_{j}\right)\right)<2 \bmod \left(A_{d}\left(z_{j}\right)\right) \text { if } z_{j} \text { is semi-critical. }
\end{aligned}
$$

In the critical case above, we say that $A_{d}\left(z_{j}\right)$ is a child to $A_{d-1}\left(z_{j+1}\right)$, i.e. when $f_{c}: A_{d}\left(z_{j}\right) \rightarrow A_{d-1}\left(z_{j+1}\right)$ is a double covering. The child is excellent if it has two other children.

Now we are ready to define the tableau.

Definition 2.3. The tableau is associated to a starting point $z=z_{0}$ and a 2dimensional array of the non-negative numbers $\mu_{i j}=\bmod \left(A_{i}\left(z_{j}\right)\right.$ ) (with the obvious notion of being critical, semi-critical and off-critical). We mark each entry in the tableau as critical if $\mu_{i j}$ is critical, semi-critical if $\mu_{i j}$ is semi-critical and off-critical if $\mu_{i j}$ is off-critical.

A movement in the north-east direction in the tableau, from $\mu_{i j}$ to $\mu_{i-1, j+1}$, represents the action of the map $f_{c}$ on the annulus $A_{i}\left(z_{j}\right)$. It follows from the definition that each column in the tableau starts with critical entries, then at some point comes a semi-critical entry, and below this only off-critical entries exist. These entries are usually depicted as single lines (critical marking), double lines (semicritical marking), and no lines (off-critical marking).

We will, from now on, only consider the critical tableau, i.e. when $z_{0}=c_{0}=0$. Write $c_{0}=0, c_{1}=c, c_{n}=f_{c}\left(c_{n-1}\right)$. We say that the critical tableau is recurrent if

$$
\sup \left\{d: \mu_{d k} \text { is critical }\right\}=\infty .
$$

We say that the critical tableau is periodic if some $k$ th column, $k>0$, is entirely critical. 
Theorem 2.4 (Yoccoz). Assume that the critical tableau is recurrent but not periodic and that there exists some non-degenerate annulus $A_{d}(0)$ such that $\bmod \left(A_{d}(0)\right)>0$. Then

$$
\sum_{d} A_{d}(0)=\infty
$$

This result depends essentially on the following two facts.

Lemma 2.5. Let $\mu_{i j}$ be a critical tableau which is recurrent but not periodic.

(1) Assume there is a child $\mu_{d 0}=A_{d}(0)$ which is excellent. Then all children to $A_{d}(0)$ are excellent.

(2) There exists at least one child $A_{d}(0)$ which is excellent in the tableau.

If $f_{c}$ is non-renormalisable, then some $A_{d}(0)$ is an excellent child and all this child's descendants are all excellent. If $A_{d^{\prime}}(0)$ is a child to $A_{d}(0)$, we have $\bmod \left(A_{d}(0)\right)=2 \bmod \left(A_{d^{\prime}}\right)$, because $f_{c}: A_{d^{\prime}}(0) \rightarrow A_{d}(0)$ is a double unbranched covering. Since every excellent child has at least 2 children, and the modulus of each of these children is half of their parents, we get that the total sum of the moduli is

$$
\sum_{A_{d^{\prime}}(0) \text { is a descendant to } A_{d}(0)} A_{d^{\prime}}(0) \geq \sum_{k \geq 0} 2^{k} \frac{1}{2^{k}} \quad \bmod \left(A_{d}(0)\right)=\infty,
$$

given that the top child $A_{d}(0)$ is non-degenerate, i.e. $\bmod \left(A_{d}(0)\right)>0$. The existence of such a non-degenerate child is automatic as soon as some $A_{n}(0)$ is nondegenerate.

Hence Yoccoz's result follows from Lemma 2.5 given the non-degeneracy condition. But what happens if $A_{d}(0)$ and all its children are degenerate? The main result of the paper is that the puzzle pieces still shrink to points.

\section{The COMPlementary annuli and proof of Theorem A}

From the previous section we have seen that the assumptions in Theorem $\mathrm{A}$ are natural and come from the construction of the Yoccoz puzzle. In particular the sequence of annuli $A_{j}$ is simply the set of descendants of an excellent child in the puzzle. Now let us prove Theorem $\mathrm{A}$.

Assume $A=A_{0}$ is a degenerate critical annulus and that $A_{j}, j=0, \ldots$ satisfies the assumptions of the theorem. Hence every child $A_{j}$ has at least two children, each mapped onto $A_{j}$ as an unbranched covering of degree 2 . Let us relabel these annuli. They form a tree of descendants $A_{i, j}$ starting from $A=A_{0,1}$ so that, for fixed $i>0$, $A_{i, j}$ are the descendants of generation $i$. Generation $i$ means that $f^{i}\left(A_{i, j}\right)=A_{0}$ and that $f^{k}: A_{i, j} \rightarrow A_{0}$ is a $2^{i}$ degree unbranched covering. Moreover, since every $A_{i, j}$ is excellent, there are at least $2^{i}$ annuli of generation $i$.

A complementary annulus $\alpha_{j}$ is defined by the annulus bounded by $A_{j}$ and $A_{j+2}$. The annulus $A_{j+1}$ is called the middle annulus (between $A_{j}$ and $A_{j+2}$ ) of $\alpha_{j}$. Of course these annuli overlap unless we take every second annulus. We will deal with this later.

In this proof we always assume that a complementary annulus is non-degenerate. In the applications it turns out that they are. We want to see what kind of relation there is between $A_{j}$ and the $\alpha_{j}$. 
Take some complementary $\alpha$ bounded by the degenerate annuli $P$ and $Q$, where $P$ surrounds $Q$. Note that we assume that exactly one annulus $R$ lies between $P$ and $Q$. Now $Q$ has a child, say $Q_{1}$, so that $Q_{1}$ maps onto $Q$ as a 2 degree unbranched covering. We want to pull back $P$ along the same branch (if possible) as $Q$, back to some $P_{j}$ surrounding $Q_{1}$.

In the first steps $\alpha$ (between $P$ and $Q$ ) is pulled back as a one-to-one map until some preimage $P_{1}$ of $P$ under $f^{k}$ surrounds the critical point $z_{0}$. This means by definition that this preimage $P_{1}$ is a child to $P$. If moreover $Q_{1}$, being the preimage of $Q$ under $f^{k}$ surrounded by $P_{1}$, also surrounds the critical point, we are done and have found $P_{1}$ surrounding $Q_{1}$, both being children of $P$ and $Q$ respectively. Clearly, if exactly one degenerate annulus lies between $P$ and $Q$ then exactly one degenerate annulus lies between $P_{1}$ and $Q_{1}$.

The second (and most probable) case is that, whereas $P_{1}$ surrounds the critical point, $Q_{1}$ does not surround the critical point. Hence we are in a semi-critical situation, so the pullback $f^{-k}(\alpha)$ is not an annulus. However, if we consider the annulus $\beta_{1}$ between $P_{1}$ and $Q_{1}$, this annulus has modulus at least $1 / 2$ of the modulus of $\alpha$ (by standard inspection from semi-critical annuli). Continuing pulling back $\beta_{1}$, we again sooner or later reach the same situation: Some pullback $P_{2}$ of $P_{1}$ under $f^{k_{1}}$ surrounds the critical point. If again the preimage $Q_{2}$ (being a preimage of $Q_{1}$ under $f^{k_{1}}$ ) surrounded by $P_{2}$ also surrounds the critical point, we are done and have found two descendants, $P_{2}$ and $Q_{2}$, to $P$ and $Q$ respectively. However, note that, whereas $Q_{2}$ is a child to $Q$, we have that $P_{2}$ is a child of $P_{1}$ and $P_{1}$ is a child of $P$. $\left(Q_{1}\right.$ is not a child of $Q$ since $Q_{1}$ was assumed not to surround the critical point.)

Continuing in this way we find two descendants $P_{m}$ and $Q_{m}$ such that

$$
f^{k+k_{1}+\ldots+k_{m-1}}: P_{m} \rightarrow P
$$

as a $2^{m}$ degree unbranched covering and

$$
f^{k+k_{1}+\ldots+k_{m-1}}: Q_{m} \rightarrow Q
$$

as a 2 degree unbranched covering.

In the same way, the middle annulus $R$ between $P$ and $Q$ is pulled back to some $R_{m}$ between $P_{m}$ and $Q_{m}$ and

$$
f^{k+k_{1}+\ldots+k_{m-1}}: R_{m} \rightarrow R,
$$

as a $D$ degree unbranched covering where $2 \leq D \leq 2^{m}$.

Here $Q_{m}$ is a child to $Q$, whereas every $P_{j+1}$ is a child to $P_{j}, j=0, \ldots, m-1$. The annulus $R_{j+1}$ is a child to $R_{j}$ only if $R_{j+1}$ surrounds the critical point.

We call the annulus bounded by $P_{m}$ and $Q_{m}$ an offspring to $\alpha$, and $\alpha$ the ancestor to the annulus between $P_{m}$ and $Q_{m}$. Hence every offspring has modulus at least $2^{-m}$ times the modulus of its ancestor $\alpha$, where $m$ is defined above.

Obviously, if there is a degenerate annulus $A$ between $P_{m}$ and $Q_{m}$, we can map this annulus forward; $f^{k+k_{1}+\ldots+k_{m-1}}(A)$ will in that case be some degenerate annulus between $P$ and $Q$.

Conversely, let $P_{m}$ and $Q_{m}$ be given degenerate annuli bounding the complementary annulus $\alpha_{1}$ and assume that exactly one degenerate annulus $R_{m}$ lies between $P_{m}$ and $Q_{m}$. If $Q_{m}$ has generation more than 1, then the parent $Q$ would have generation at most 1 . On the other hand, the parent $P$ to $P_{1}$, which in turn is parent to $P_{2}$ and so on down to $P_{m}$, might have negative generation, meaning that 
$P$ is actually a parent to $A_{0}$. In this case, $A_{0}$ would lie between $P$ and $R$. But in this case there has to be some preimage $A_{j}^{\prime}$ of $A_{0}$ laying between $P_{m}$ and $Q_{m}$. If also $R$ has negative generation, then there is some preimage to $A_{0}$ different from $R_{m}$ between $Q_{m}$ and $R_{m}$; a contradiction. Hence $R$ has generation at most zero.

If the generation of $R$ is greater than zero, then the generation of $P$ has to be zero, i.e. $P=A_{0}$, because otherwise we could pull back $A_{0}$ to some degenerate annulus between $R_{m}$ and $P_{m}$.

From this we conclude:

Fact. If there is exactly one degenerate annulus between $Q_{m}$ and $P_{m}$, then there is exactly one degenerate annulus between $Q$ and $P$, if the generation of $R$ is at least 1.

Definition 3.1. Given a complementary annulus $\alpha$ bounded by $P_{m}$ and $Q_{m}$, with middle annulus $R_{m}$, we say that its intermediate generation is equal to the generation of $R$, where $R=f^{k+k_{1}+\ldots+k_{m-1}}\left(R_{m}\right)$ and $f^{k+k_{1}+\ldots+k_{m-1}}$ is the function described above.

From the discussion we conclude:

Lemma 3.2. Every complementary annulus $\alpha$ with intermediate generation at least 1 has some unique ancestor $\beta$.

Proof. Clearly, the annulus $P=f^{k+k_{1}+\ldots+k_{m-1}}\left(P_{m}\right)$ must have generation at most zero. That means that no denegerate annulus can exist between $P$ and $R$ or between $R$ and $Q$ because this would then have a preimage $R^{\prime}$ between $Q_{m}$ and $P_{m}$ different from $R$; a contradiction. Hence the annulus $\beta$ bounded by $P$ and $Q$ is indeed a complementary annulus.

Definition 3.3. Given a complementary annulus $\alpha$ bounded by the outer degenerate annulus $A_{m, *}$ and the inner degenerate annulus $A_{n, *}$, we say that the outer generation to $\alpha$ is equal to $m$ and the inner generation to $\alpha$ is $n$. We write $\alpha=\alpha_{n, *}^{m}$, where $*$ means an index, since there might be many $\alpha$ with the same $m$ and $n$.

We have proved the following.

Lemma 3.4. For every complementary annulus $\alpha=\alpha_{n, *}^{m}$ with intermediate generation at least 1 and with ancestor $\alpha_{n-1, *}^{m_{1}}$ we have

$$
\bmod \left(\alpha_{n, *}^{m}\right) \geq 2^{m_{1}-m} \bmod \left(\alpha_{n-1, *}^{m_{1}}\right) .
$$

Corollary 3.5. For every complementray annulus $\alpha_{n, *}^{m}, n>1$ with intermediate generation at least 1 , there is some grand ancestor $\alpha_{N, *}^{m_{n-1}}$ such that

$$
\bmod \left(\alpha_{n, *}^{m}\right) \geq 2^{m_{n-N}-m} \bmod \left(\alpha_{N, *}^{m_{n-N}}\right) .
$$

Since the number of degenerate annuli of generation $m$ is at least $2^{m}$, we have that the number of complementary annuli of outer generation $m$ is at least $2^{m}$. If the complementary annuli are non-degenerate, there is some $M_{0}>0$, such that $\bmod \left(\alpha_{N, *}^{m}\right) \geq M_{0}$ for all grand ancestors $\alpha_{N, *}^{m}$.

When we consider the complementary annuli, we want to sum every second moduli (since otherwise they overlap). Clearly, the set of complementary annuli which have intermediate generation at most 0 are finite. Therefore, we can fix a generation $m_{0}>1$ such that all $\alpha_{n, *}^{m}, m \geq m_{0}$, have intermediate generation at least 1 . So every such annulus has some grand ancestor. 
Now consider the set of the annuli $\alpha_{n, *}^{m_{0}}$. Either half of them will have the property that the inner annulus is $A_{j}$, where $j$ is even, or at least half of them will have an inner annulus being $A_{j}$ where $j$ is odd. Suppose that the first case occurs. In this collection of complementary annuli, all the inner generations are even so they do not overlap. We get

$$
\sum_{n, *} \bmod \left(\alpha_{n, *}^{m_{0}}\right) \geq 2^{m_{0}-1} 2^{m_{n-N}-m_{0}} \bmod \left(\alpha_{N, *}^{m_{n-N}}\right) \geq M_{0} / 2,
$$

where the sum runs over the annuli $\alpha_{n, *}^{m_{0}}$, where all of them have inner generation even. Of course the same statement holds in the odd case.

Going sufficiently deep in the nest we pick another $m_{1}>m_{0}$ such that no $\alpha_{n, *}^{m_{1}}$ intersects any $\alpha_{n, *}^{m_{0}}$. Again, the sum of these annuli which do not overlap becomes at least $M_{0} / 2$. Continuing in this manner we get a sequence $m_{0} \leq m_{1} \leq m_{2} \ldots$ of outer generations such that the sum of non-overlaping complementary annuli of generations $m_{0}, m_{1}, \ldots$ becomes at least

$$
M_{0} / 2+M_{0} / 2+\ldots=\infty
$$

and Theorem $\mathrm{A}$ follows.

\section{THE NON-DEGENERACY OF THE COMPLEMENTARY ANNULI}

So far we have seen that degenerate annuli in the Yoccoz puzzle is not an obstacle to prove that puzzle pieces shrink to points, under the condition that the complementary annuli are non-degenerate. In the applications, the combinatorics come from the Mandelbrot set $\mathcal{M}$ and it turns out that the complementary annuli are automatically non-degenerate.

Given a non-renormalisable quadratic polynomial $f_{c}(z)=z^{2}+c$, the schematic picture of the first two levels of the Yoccoz puzzle is shown in Figure 1 (here we have chosen $c$ not from the 1/2-limb of the Mandelbrot set).
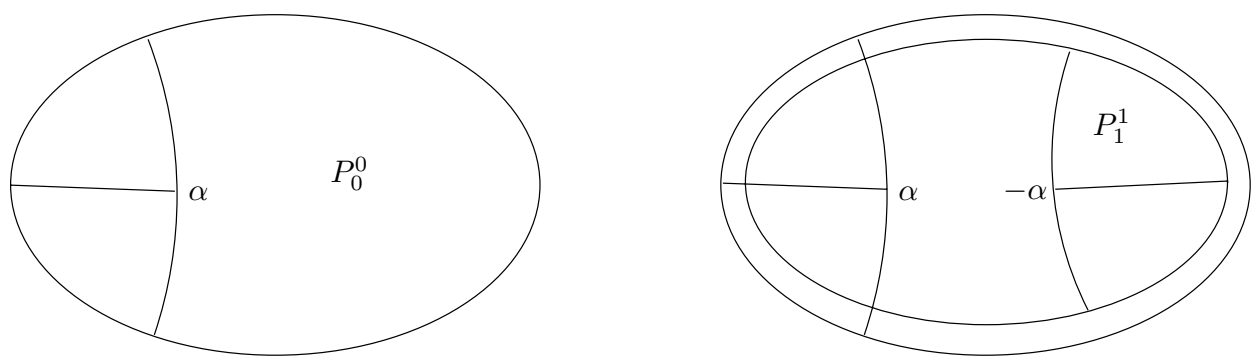

Figure 1. The Yoccoz puzzles of depths 0 and 1 , with $q=3$ external rays landing at $\alpha$. The ellipse to the left is an equipotential and the inner ellipse to the right is its preimage.

Generally, puzzle pieces containing the critical point are mapped onto each other under iterations of $f^{q}$, where $q \geq 2$, i.e. at least 2 iterates of $f$ is required for some critical puzzle piece $P_{d}^{0}$ to be mapped onto another critical puzzle piece $P_{d^{\prime}}^{0}$ (in the figure $q=3$ ). Hence, between $P_{d}^{0}$ and $P_{d^{\prime}}^{0}$ there is at least one puzzle piece 
containing $P_{d^{\prime}}^{0}$ and contained in $P_{d}^{0}$. If, now, the two top puzzle pieces $P_{1}^{1}$ and $P_{0}^{0}$ touch each other at some set $E$, the set $E$ will be pulled back and produce degeneracies at all depths between preimages of $P_{1}^{1}$ and $P_{0}^{0}$. However, these touching points cannot coincide and we have the following:

Fact. For any depth $d \geq 0$ and nested puzzle pieces $P_{d} \supset P_{d+1} \supset P_{d+2}$ we have that $P_{d+2} \Subset P_{d}$, i.e. there are no touching points between $P_{d}$ and $P_{d+2}$.

So if we go two levels down in the nest we create a non-degenerate annulus. Since we always go at least 2 levels down between consecutive critical puzzle pieces, the complementary annuli $\alpha_{d}$ being the space between $A_{d}(0)$ and its pullback to one if its grandchildren $A_{d^{\prime}}(0), d^{\prime} \geq d+2 q \geq d+4$, have to be non-degenerate, i.e. $\bmod \left(\alpha_{d}\right)>0$.

Note that if $c$ belonged to the $1 / 2$-limb of $\mathcal{M}$, then the annulus between $A_{j}$ and $A_{j+1}$ could be degenerate also. This is the reason why we consider the complementary annuli being the space between every second annulus in the $a$-nested sequence $A_{j}, j=0, \ldots$

Hence for all non-renormalisable combinatorics from the Mandelbrot set, Theorem $\mathrm{A}$ works. In particular, the puzzle pieces in the Yoccoz puzzle $P_{d}\left(z_{0}\right)=$ in $\left(A_{d-1}\left(z_{0}\right)\right)$ containing the critical point $z_{0}$ must shrink to a single point:

$$
\bigcap_{j \geq 0} i n\left(A_{j}\right)=\left\{z_{0}\right\}
$$

\section{ACKNOWLEDGEMENTS}

I thank Carsten Petersen for discussions which led to this paper. I am thankful to M. Yampolsky for discussing this problem and giving helpful remarks. The paper was written at Mathematisches Seminar, Christian-Albrechts Universität zu Kiel. I gratefully acknowledge the hospitality of the department.

\section{REFERENCES}

1. Magnus Aspenberg and Michael Yampolsky. Mating non-renormalisable quadratic polynomials. Comm. Math. Phys. 287(1):1-40, 2009.

2. Bodil Branner and John Hubbard. The iteration of cubic polynomials II. Patterns and parapatterns. Acta. Math., 169(3-4):229-325, 1992. MR.1194004 (94d:30044)

3. J. H. Hubbard. Local connectivity of Julia sets and bifurcation loci: three theorems of J.C. Yoccoz. In Topological methods in modern mathematics (Stony Brook, NY, 1991), pages 467-511. Publish or Perish, Houston, TX, 1993. MR.1215974 (94c:58172)

4. Jeremy Kahn and Michael Lyubich. A priori bounds for some infinitely renormalizable quadratics: I. Bounded primitive combinatorics. Preprint, math.DS/0609045.

5. Jeremy Kahn and Michael Lyubich. A priori bounds for some infinitely renormalizable quadratics: II. Decorations. Preprint, math.DS/0609046.

6. Mikhail Lyubich. Dynamics of quadratic polynomials. I, II. Acta Math., 178(2):185-247, 247297, 1997. MR1459261 (98e:58145)

7. John Milnor. Local connectivity of Julia sets: expository lectures. In The Mandelbrot set, theme and variations, volume 274 of London Math. Soc. Lecture Note Ser., pages 67-116. Cambridge Univ. Press, Cambridge, 2000. MR.1765085 (2001b:37073)

8. John Milnor. Dynamics in one complex variable, volume 160 of Annals of Mathematics Studies. Princeton University Press, Princeton, NJ, third edition, 2006. MR2193309 (2006g:37070)

9. Pascale Roesch. Cubic polynomials with a parabolic point. Preprint. arXiv:0712.3372. 
10. Pascale Roesch. Hyperbolic components of polynomials with a fixed critical point of maximal order. Ann. Sci. École Norm. Sup. (4), 40(6):901-949, 2007. MR2419853 (2009c:37042)

11. Pascale Roesch and Yongcheng Yin. The boundary of bounded polynomial Fatou components. C. R. Math. Acad. Sci. Paris, 346 (15-16):877-880, 2008. MR2441925

Mathematisches Seminar, Christian-Albrechts Universität zu Kiel, Ludewig-Meyn Str.4, 24098 Kiel, Germany

E-mail address: aspenberg@math.uni-kiel.de

E-mail address: maspenberg@gmail.com 